ARTIGO ORIGINAL

\title{
CONTAMINAÇÃO SAZONAL POR OVOS DE HELMINTOS \\ NA PRAIA DE IPANEMA, EM PORTO ALEGRE, \\ RIO GRANDE DO SUL, BRASIL
}

Viviana Cauduro Matesco, ${ }^{1}$ Márcia Bohrer Mentz, ${ }^{2}$ Marilise Brittes Rott ${ }^{2} e$ Caroline de Oliveira Silveira ${ }^{3}$

\section{RESUMO}

Com o objetivo de estudar a freqüência de ovos de helmintos, de interesse da saúde pública, e a sazonalidade da contaminação no ambiente, foram coletadas amostras de areia e de fezes de animais no período de março de 2004 a fevereiro de 2005, na praia de Ipanema, no município de Porto Alegre (RS), Brasil. Em laboratório, as amostras de areia foram submetidas às técnicas de Faust modificada e Kazacos modificada; as amostras de fezes, à técnica de Willis. Das 60 amostras de areia, $8(13,3 \%)$ foram positivas para ovos dos seguintes parasitos: Ascaris (8,3\%), Toxocara spp. $(3,3 \%)$ e "ancilostomídeo like" (1,7\%). Das 121 amostras de fezes, 39 (33,9\%) foram positivas para ovos dos gêneros: Ancylostoma (29,8\%), Trichuris (3,3\%) e Toxocara $(0,8 \%)$. No mês de junho foi encontrado o maior índice de contaminação nas amostras de areia, enquanto as amostras de fezes de animais revelaram contaminação relativamente uniforme ao longo do ano. Embora os resultados não revelem freqüências elevadas de formas parasitárias nas amostras de areia e de fezes, recomenda-se a adoção de medidas preventivas que promovam a saúde humana e animal.

DESCRITORES: Helmintos. Areia. Fezes. Saúde pública. Zoonoses. Sazonalidade.

\section{INTRODUÇÃO}

Animais de companhia desempenham importante papel nas sociedades, contribuindo para o desenvolvimento físico, social e emocional de crianças e para o bem-estar de seus donos (16). Entretanto, cães e gatos possuem muitos parasitos próprios, cujas larvas infectantes só completam seu ciclo nesses hospedeiros.

1 Bacharel do curso de Ciências Biológicas - Ênfase Ambiental, Instituto de Biociências, Universidade Federal do Rio Grande do Sul (UFRS), Porto Alegre-RS, Brasil.

2 Setor de Parasitologia do Departamento de Microbiologia do Instituto de Ciências Básicas da Saúde, UFRS, Porto Alegre-RS, Brasil.

3 Aluna da Faculdade de Farmácia, UFRS, Porto Alegre-RS, Brasil.

Endereço para correspondência: Márcia Bohrer Mentz, Rua Fernandes Vieira, 350, ap. 1101, Bairro Bom Fim. CEP 90035-090, Porto Alegre, Rio Grande so Sul, Brasil. E-mail: mbmentz@uol.com.br

Recebido para publicação em 18/10/2005. Revisto em 13/5/2006. Aceito em 20/7/2006. 
Ao infectar acidentalmente o ser humano, as larvas da maioria das espécies não são capazes de se desenvolver, mas podem migrar para os tecidos subcutâneo ou visceral e causar, no primeiro, a síndrome conhecida como larva migrans cutânea e, no segundo, as síndromes denominadas larva migrans visceral e larva migrans ocular. Os agentes etiológicos são larvas infectantes do gênero Ancylostoma, entre outras espécies, e ovos larvados de Toxocara spp. $(3,9,15)$.

O número crescente de cães e gatos com acesso a áreas de lazer, os quais depositam suas fezes em locais freqüentados pela população, especialmente nos centros urbanos, torna alta a expectativa de contaminação do solo com ovos de helmintos $(7,10,18,20)$.

No município de Porto Alegre (RS), a praia de Ipanema figura entre os locais públicos freqüentemente utilizados pela população para lazer e recreação. Nesse local encontram-se os fatores epidemiológicos necessários para a ocorrência de doenças parasitárias transmitidas por animais: condições climáticas favoráveis para a fase de vida livre dos parasitos e um grande número de cães, errantes e com dono, que transitam livremente nas vias de passeio para pedestres e na areia da praia. Considerando a ausência de informações sobre o nível de contaminação da área, este estudo teve como objetivos verificar a freqüência de ovos de helmintos, de interesse da saúde pública, na areia da praia de Ipanema e nas fezes de animais depositadas no local, assim como a sazonalidade da contaminação ambiental.

\section{MATERIAL E MÉTODOS}

As coletas de amostras de areia e fezes foram realizadas mensalmente, entre março de 2004 e fevereiro de 2005, na praia de Ipanema situada à beira do estuário Guaíba, no bairro Ipanema, em Porto Alegre (RS). Esta faixa arenosa de cerca de mil metros de extensão sofre variações conforme o nível hídrico, predominando a cobertura arenosa de granulação fina que acompanha um calçadão para pedestres. É utilizada como balneário nos meses de verão pela população de baixa renda, apesar de suas águas terem sido classificadas como impróprias para o banho.

Amostras de areia

Para a amostragem da areia, foram estabelecidos cinco pontos ao longo da faixa arenosa, com distância regular aproximada de $200 \mathrm{~m}$ entre eles, os quais foram utilizados em todas as coletas. Em cada ponto, foi definido um quadrante de $2 \mathrm{~m}$ de lado, onde foram coletados cerca de $50 \mathrm{~g}$ de areia em cada um dos quatro vértices, por raspagem superficial e profunda $(5 \mathrm{~cm})$ do solo, utilizando-se colheres plásticas. Cada amostra, totalizando aproximadamente $200 \mathrm{~g}$ de areia, foi acondicionada em um saco plástico e etiquetada. Repetiu-se o mesmo procedimento nos demais pontos de coleta. Em seguida, as amostras eram transportadas ao Laboratório de Parasitologia da UFRGS, em caixa de isopor, e mantidas sob refrigeração até 
seu processamento. Para a recuperação dos ovos de helmintos, cada amostra foi submetida a duas técnicas: Faust (5) modificada em laboratório, segundo Mentz et al. (10), e Kazacos (8). Esta última também sofreu modificações, brevemente descritas a seguir: cada sub-amostra de $30 \mathrm{~g}$ foi homogeneizada em $40 \mathrm{ml}$ de solução de Tween 20 a 0,5\%, em copo plástico, com auxílio de bastão de vidro. No passo seguinte, quatro sub-amostras foram filtradas através de gaze para um tubo plástico de centrífuga (capacidade $30 \mathrm{ml}$; diâmetro $25 \mathrm{~mm}$ ) e centrifugadas em $500 \times \mathrm{g}$ durante três minutos. Depois de desprezado o sobrenadante, foram adicionados $20 \mathrm{ml}$ de água. A etapa de centrifugação, seguida da lavagem, foi repetida duas vezes. O sobrenadante foi, então, outra vez desprezado e foram adicionados $16 \mathrm{ml}$ de solução de sulfato de zinco (densidade 1:35), após o que foi feita a ressuspensão do sedimento e a centrifugação a $500 \mathrm{x} g$ por dois minutos. Os tubos foram retirados da centrífuga, completando-se o volume com solução de sulfato de zinco (1:35) até a formação de um menisco invertido. Colocou-se lâmina de microscopia ( $25 \times 75 \mathrm{~mm})$ e, ao fim de 15 minutos, acrescentou-se uma lamínula $(24 \times 24 \mathrm{~mm})$ para exame no microscópio óptico (100X) e pesquisa de elementos parasitários.

Amostras fecais

As amostras de fezes de animais foram coletadas ao longo de toda a extensão da faixa arenosa. As amostras de fezes, com aproximadamente $10 \mathrm{~g}$ cada uma, foram coletadas com o auxílio de palitos de madeira e acondicionadas em potes plásticos descartáveis com tampa, devidamente identificados. Para sua conservação o material fecal foi submetido à homogeneização com $2 / 3$ de formol $10 \%$ e mantido a, aproximadamente, $4^{\circ} \mathrm{C}$ em laboratório.

Para o processamento, optou-se pela técnica de Willis (21), com a utilização de solução hipersaturada de cloreto de sódio $(\mathrm{NaCl})$. As lâminas foram examinadas no microscópio óptico (100X) para pesquisa de elementos parasitários.

\section{RESULTADOS E DISCUSSÃO}

Das 60 amostras de areia coletadas na praia de Ipanema, entre março de 2004 e fevereiro de 2005, 13,3\% (oito amostras) apresentaram-se positivas para ovos de helmintos; enquanto nas 121 amostras de fezes de animais coletadas no mesmo período, o percentual de positividade atingiu 33,9\% (39 amostras), conforme a Figura 1. Outros estudos também evidenciaram maior prevalência de parasitos em amostras de fezes do que nas de solo $(13,14)$. Nas fezes, ocorre a concentração de um grande número de elementos parasitários; no meio ambiente, porém, elas são desagregadas pela ação mecânica de pisoteio, chuvas, ventos ou vetores, o que acarreta a disseminação ou mesmo a destruição dos ovos de parasitos (7).

Até o presente momento, em poucos estudos avaliou-se a freqüência de contaminação por ovos de helmintos em praias $(11,12,19)$. Entretanto, áreas 
próximas a mananciais hídricos parecem apresentar níveis de contaminação mais baixos que parques e praças públicas de zonas urbanas (12). A diferença das praias em relação a outros locais se deve, possivelmente, ao fato de que essas áreas são espaços abertos, expostos às intempéries e suscetíveis à ação antrópica $(11,20)$.

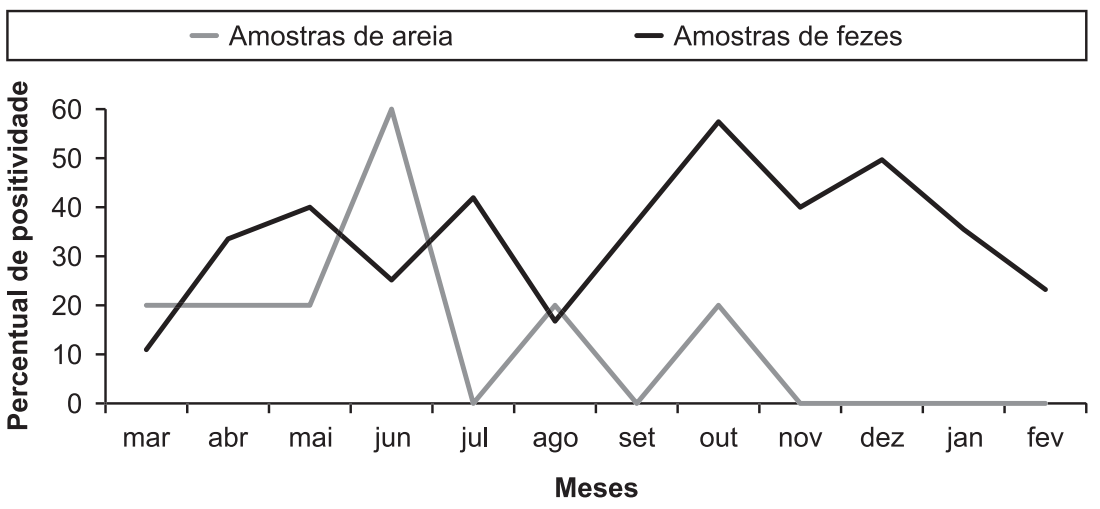

Figura 1. Distribuição mensal (março/2004 a fevereiro/2005) do percentual de amostras de areia e de fezes positivas para formas parasitárias na praia de Ipanema, Porto Alegre (RS), Brasil.

Nas praias de Corrientes (Argentina), parasitos zoonóticos foram identificados em 32,7\% das amostras de areia e 59,3\% das fezes (11); no Balneário Cassino (Brasil), do total de amostras de fezes caninas coletadas, $86,1 \%$ apresentaram positividade para ovos e/ou larvas de helmintos (19), valores superiores aos encontrados neste estudo.

Em decorrência de sua extensão, depreende-se que a praia de Ipanema apresenta amplas possibilidades de recuperação. A habilidade de recuperação do solo depende de sua estrutura física e textura, bem como das condições biológicas que influenciam esse processo. A dessecação pelos raios solares e a lavagem dos ovos pela chuva, que os arrasta para camadas mais profundas, são os principais fatores responsáveis pela purificação do solo (12).

Em relação aos parasitos encontrados, os ovos de "ancilostomídeo like" apareceram com baixa freqüência em amostras de areia (1,7\%), embora Ancylostoma spp. tenha sido o parasito prevalente nas amostras de fezes (29,8\%), o que está de acordo com resultados encontrados em fezes caninas do balneário Cassino (19). Outros autores também registraram resultados semelhantes em amostras de solo em áreas urbanas $(2,4,10)$. Isso pode ser explicado pelo fato de que as larvas deixam os ovos ainda no primeiro estádio no período de 24 a 48 horas, passando a não ser detectadas pelas técnicas de rotina (1). Assim, Nunes et al. (13), empregando técnicas distintas para a recuperação de ovos e de larvas em amostras 
de solo, encontraram percentual de apenas $0,56 \%$ para ovos, enquanto as larvas atingiram positividade de $46,4 \%$.

Ovos de Ascaris foram os mais freqüentes nas amostras de areia da praia estudada $(8,3 \%)$, porém ocorreram em menor proporção quando comparados aos resultados encontrados em caixas de areia de parques da cidade de Porto Alegre (RS) $(16,7 \%)(10)$.

No presente estudo, a freqüência de Toxocara spp. nas amostras de areia $(3,3 \%)$ e de fezes $(0,8 \%)$ foi semelhante aos resultados registrados nas praias de Corrientes, onde $T$. canis foi o terceiro parasito mais prevalente em fezes caninas $(4,1 \%)$ e o segundo mais prevalente em areia $(0,3 \%)(11)$.

Ovos de Trichuris spp. não foram encontrados na areia da praia de Ipanema, sendo raros os relatos de sua presença em amostras de solo $(4,12)$. No entanto, foi o segundo parasito mais freqüente nas amostras de fezes $(3,3 \%)$, o que é corroborado pelo achado de Scaini et al. (19).

Poucos autores avaliaram a variação sazonal na freqüência de ovos de parasitos. Em regiões de clima temperado, o efeito da sazonalidade nas taxas de transmissão está relacionado à variação de temperatura e umidade. Em regiões tropicais, porém, temperatura e umidade parecem não ser suficientes para determinar um decréscimo significativo na taxa de transmissão da maioria dos enteroparasitos (14). Santarém et al. (18) verificaram maior recuperação de ovos de Toxocara spp. nos meses da primavera e do verão. Sommerfelt et al. (20) e Salinas et al. (17) obtiveram valores semelhantes para contaminação do solo com ovos de T. canis no outono e na primavera, porém menores no inverno e no verão.

No presente estudo, a média de contaminação mensal foi de 0,7 amostras positivas de areia. Em junho ocorreu o maior número de amostras positivas (três) e nos meses de julho, setembro e novembro a fevereiro não se verificou a presença de ovos (Figura 1). Em amostras de fezes, o percentual mensal médio de contaminação foi de $34,2 \%$. Outubro foi o mês com maior percentual de positividade $(57,1 \%) \mathrm{e}$ março, o mês com menor percentual (11,1\%) (Figura 1).

Os resultados obtidos na praia de Ipanema concordam com aqueles de Habluetzel et al. (7), que constataram maiores níveis de contaminação ambiental por Toxocara sp. nos meses mais frios do ano.

A análise das amostras de fezes revelou contaminação relativamente uniforme ao longo do ano (Figura 1).

Os resultados observados neste estudo mostraram elevada prevalência de ovos de Ancylostoma spp. nas fezes e baixa prevalência de ovos de Toxocara spp. em areia e fezes coletadas na praia de Ipanema. Isso indica um risco maior de aquisição de larva migrans cutânea (LMC) que de larva migrans visceral (LMV).

O movimento constante na área estudada e a ação da fauna local favorecem a desintegração do material fecal e sua mistura com a areia, dificultando a posterior visualização e remoção das fezes. Esse fator, somado aos aspectos biológicos dos helmintos - que requerem entre uma e três semanas de maturação 
- configuram forte argumento em favor de um controle diário das fezes $(1,11$, $12,15)$. Além disso, são necessários programas de educação sanitária que tornem possível a compreensão do potencial zoonótico das parasitoses transmitidas através do solo e estimulem, entre os donos de animais domésticos, o desenvolvimento do conceito de posse responsável. Este inclui a consciência da necessidade de controle da população canina e felina $(6,16)$.

\section{ABSTRACT}

Seasonal environment contamination by helminth eggs in Ipanema Beach, Porto Alegre, Rio Grande do Sul, Brazil

The study analyzed 60 sand samples and 121 animal faecal samples collected in Ipanema Beach, Porto Alegre city, RS, Brazil, from March, 2004 to February, 2005. The laboratory techniques employed were: modified Faust and modified Kazacos for sand samples and Willis' technique for feces. The results point out the presence of parasites in $13,3 \%$ of the sand samples: Ascaris $(8,3 \%)$, Toxocara spp. $(3,3 \%)$ and ancilostomid like (1,7\%). Nematode eggs were found in 33,9\% of the faecal samples: Ancylostoma spp. (29,8\%), Trichuris spp. (3,3\%) and Toxocara sp. (0,8\%). Higher contamination levels in sand samples occurred in June, while uniform contamination throughout the year was observed in faecal samples. In spite of the low frequency of parasitic forms in sand and faecal samples, we recommend the adoption of broad preventive measures promoting human and animal health.

KEYWORDS: Parasites. Sand. Fecal samples. Public health. Zoonosis. Sazonality.

\section{REFERÊNCIAS}

1. Acha P, Szyfres B. Zoonosis y enfermedades transmisibles comunes al hombre y a los animales. 2. ed. OMS/OPS. Washington, 1986.

2. Alcântara N, Bavia E, Silvão RM, Carvalho E. Environmental contamination by Toxocara sp. eggs in public areas of Salvador, Bahia State, Brazil. Rev Soc Bras Med Trop 22: 187-190, 1989.

3. Anaruma-Filho F, Chieffi PP, Correa CRS, Camargo ED, Silveira EPR, Aranha JJB, Ribeiro MCSA. Human toxocariasis: a seroepidemiological survey in the municipality of Campinas (SP), Brazil. Rev Inst Med Trop São Paulo 44: 303-307, 2002.

4. Chieffi PP, Müller EE. Prevalência de parasitismo por Toxocara canis em cães e presença de ovos de Toxocara sp. no solo de localidades públicas da zona urbana do Município de Londrina, Estado do Paraná, Brasil. Rev Saúde Pública 10: 367-372, 1976.

5. Faust EC, D’Antoni JS, Odom V, Miller MJ, Peres C, Sawitz W, Thomen LF, Tobbie J, Walkern JH. A critical study of clinical laboratory technics for the diagnosis of protozoan cysts and helminth eggs in feces. I. Perliminary communication. Am J Trop Med 18: 169-183, 1938.

6. Fonrouge R, Guardis MV, Radman NE, Archelli SM. Contaminación de suelos con huevos de Toxocara sp. en plazas y parques públicos de la ciudad de La Plata, Buenos Aires, Argentina. Bol Chil Parasitol 55: 83-85, 2000. 
7. Habluetzel A, Traldi G, Ruggieri S, Attili AR, Scuppa P, Marchetti R, Menghini G, Esposito F. An estimation of Toxocara canis prevalence in dogs, environmental egg contamination and risk of human infection in the Marche region of Italy. Vet Parasitol 113: 243-252, 2003.

8. Kazacos KR. Improved method for recovering ascarid and other helminth eggs from soil associated with epizootics and during survey studies. Am J Vet Res 44: 896-900, 1983.

9. Luz C, Nunez Rocha LF. Contaminação de localidades públicas com enteroparasitos na cidade de Goiânia-Goiás - Brasil. Rev Patol Trop 30: 235-242, 2001.

10. Mentz MB, Rott MB, Jacobsen SIV, Baldo G, Rodrigues-Júnior V. Freqüência de ovos de Toxocara spp. em três parques públicos da cidade de Porto Alegre, Rio Grande do Sul, Brasil. Rev Patol Trop 33: 105-112, 2004.

11. Milano AMF, Oscherov EB. Contaminación por parásitos caninos de importancia zoonótica en playas de la ciudad de Corrientes, Argentina. Parasitol Latinoam 57: 119-123, 2002.

12. Mizgajska $\mathrm{H}$. The role of some enviromental factors in the contamination of soil with Toxocara spp. and other geohelminth eggs. Parasitol Int 46: 67-72, 1997.

13. Nunes CM, Pena FC, Negrelli GB, Anjo CGS, Nakano MM, Stobbe NS. Ocorrência de larva migrans na areia de áreas de lazer das escolas municipais de ensino infantil, Araçatuba, SP, Brasil. Rev Saúde Pública 34: 656-658, 2000.

14. Oge H, Oge S. Quantitative comparison of various methods for detecting eggs of Toxocara canis in samples of sand. Vet Parasitol 92: 75-79, 2000.

15. Rey, L. Parasitologia Médica. Editora Guanabara Koogan S.A. Rio de Janeiro, 2001.

16. Robertson ID, Irwin PJ, Lymbery AJ, Thompson RCA. The role of companion animals in the emergence of parasitic zoonoses. Int J Parasitol 30: 1369-1377, 2000.

17. Salinas $P$, Matamala M, Schenone H. Prevalência de hallazgo de huevos de Toxocara canis en plazas de la Región Metropolitana de la ciudad de Santiago, Chile. Bol Chil Parasitol 57: 102-105, 2001.

18. Santarém VA, Sartor IF, Bergamo FMM. Contaminação, por ovos de Toxocara spp., de parques e praças públicas de Botucatu, São Paulo, Brasil. Rev Soc Bras Med Trop 31: 529-532, 1998.

19. Scaini CJ, Toledo RN, Lovatel R, Dionello MA, Gatti FA, Susin L, Signorini VRM. Contaminação ambiental por ovos e larvas de helmintos em fezes de cães na área central do Balneário Cassino, Rio Grande do Sul. Rev Soc Bras Med Trop 36: 617-619, 2003.

20. Sommerfelt I, Degregorio O, Barrera M, Gallo G. Presencia de huevos de Toxocara spp. en paseos públicos de la ciudad de Buenos Aires, Argentina, 1989-1990. Rev Med Vet 73: 70-74, 1992.

21. Willis HH. A simple levitation method for the detection of hookworm ova. Med J Australia 29: 375376, 1921. 